\title{
Asistencialismo y búsqueda de ayudas como estrategia de supervivencia en contextos campesinos clientelares ${ }^{*}$
}

\author{
Fernando Landini \\ Facultad de Psicología, Universidad de Buenos Aires, Argentina. \\ Email: landini_fer@hotmail.com
}

\begin{abstract}
Resumen: Usualmente, los estudios sobre clientelismo tienden a generar una interpretación externa del fenómeno y a dejar fuera del análisis los procesos culturales y psicosociales de carácter comunitario en que se apoyan estas prácticas. Por esta razón, se realizó un estudio de caso en una localidad campesina de la provincia de Formosa, Argentina, con el fin abordar la dimensión subjetiva del clientelismo y la búsqueda de asistencia personalizada como estrategia de supervivencia. Se concluye que las relaciones de reciprocidad entre campesinos y actores pudientes como políticos y patrones constituyen un elemento propio de la cultura campesina. No obstante, se aclara que esta práctica es mucho más amplia y no siempre se articula en términos de clientelismo político. Finalmente, se afirma que si bien el clientelismo muchas veces puede explicarse a partir de relaciones de poder, en otros casos el sentimiento de gratitud entre campesinos y patrones constituye el aspecto más destacado.
\end{abstract}

Palabras clave: clientelismo; campesinado; reciprocidad; gratitud; poder.

\section{Welfarism and seeking for social assistance as a survival strategy in peasant contexts characterized by political clientelism}

\begin{abstract}
Usually, the investigations on political clientelism tend to adopt and external point of view of the phenomenon which do not take into account the cultural and psychosocial processes that support these practices. Thus, I performed a case study in a peasant community located in the province of Formosa, Argentina, aiming to approach both, the subjective dimension of clientelism and the seeking of social assistance as a survival strategy. I conclude that the existence of relations of reciprocity between wealthy actors such as politicians and landlords, and small farmers, is characteristic of the peasant culture. Nonetheless, despite the fact it is clear that these practices are related to clientelism, they are not the same. Finally, I argue that while political clientelism sometimes can be explained focusing on relations of power, in other cases the feeling of gratitude between peasants and landlords is the most important aspect.
\end{abstract}

Key words: clientelism; peasantry; reciprocity; gratitude; power. 


\section{Assistencialismo e instrumentos de pesquisa como uma estratégia de sobrevivência em contextos de clientelismo rural}

Resumo: Normalmente, os estudos sobre o clientelismo tendem a gerar uma interpretação externa do fenômeno deixando de fora o análise dos processos culturais e psicossociais de caráter comunitário que apoiam estas práticas. Por isso, foi realizado um estudo de caso em uma vila rural na província de Formosa, na Argentina, para enfrentar a dimensão subjetiva do clientelismo eo atendimento pessoal como uma estratégia de sobrevivência. Concluímos que as relações recíprocas entre os camponeses e atores ricos como políticos e patrões são um elemento próprio da cultura rural. No entanto, é claro que esta prática é muito mais vasta e não é sempre articulada em termos de protecção política. Por fim, alega-se que o patrocínio, enquanto muitas vezes pode ser explicado por relações de poder, em outros casos, o sentimento de gratidão entre os camponeses e os padrões é o destaque.

Palavras-chave: clientelismo, gratidão, campesinato, reciprocidade.

\section{Introducción}

Es propio de los entornos culturales campesinos que aquellos pequeños productores que tienen un mal pasar esperen que los políticos locales y los productores más acaudalados se comporten de manera generosa y aporten a la satisfacción de sus necesidades en tiempos de escasez (Henningsen, 2001). Así, en estos contextos no resulta extraña la existencia de vínculos de reciprocidad desiguales donde un actor social más poderoso se compromete a brindar ayuda en tiempos de necesidad, recibiendo como contrapartida deferencia y gratitud de parte del campesino, sentimientos que podrán traducirse en distintas formas de apoyo e incluso obediencia. Se trata, ciertamente, de un entramado complejo de vínculos interpersonales, de favores recíprocos, de sentimientos personales, de formas de ejercicio del poder social y económico, y de sistemas ideológicos legitimadores que, en ciertos contextos, puedan adoptar la forma de prácticas clientelares.

Ahora bien, pese a que la figura del clientelismo político constituye una de las imágenes utilizadas con mayor frecuencia para estudiar la dinámica política en América Latina (Auyero, 1999), las prácticas culturales tradicionales que, en las sociedades rurales campesinas, se sitúan en su base, no parecen haber sido suficientemente abordadas. De hecho, los estudios y las referencias al clientelismo aparecen más en el contexto de estudios sociológicos orientados a comprender procesos macrosociales, quedando en un segundo plano la dimensión cultural y psicosocial de estas prácticas. Además, como señalan diversos autores, la mayor parte de las investigaciones sobre clientelismo lo que han hecho es generar interpreta- 
ciones del fenómeno que desconocen la mirada de los propios actores (Auyero, 1999, 2000, 2001; Trotta, 2003), por lo que aun hace falta comprender los sentidos que los mismos "clientes" dan a estas prácticas. De hecho, no incorporar el modo en que los propios actores dan sentido a sus experiencias, muchas veces lleva a acusarlos de oportunistas, lo que implica desconocer el entramado subjetivo de legitimación en el que se apoya su accionar.

De esto se sigue, entonces, que la falta de estudio, desde una perspectiva cultural y psicosocial, de los procesos, vínculos y entramados comunitarios en que se apoyan las prácticas clientelares constituye una limitación de los desarrollos académicos en torno al clientelismo. Y esto, incluso, no sólo debe ser visto como un área de vacancia científica sino como un problema concreto a la hora de diseñar y ejecutar políticas públicas asistenciales. En efecto, si coincidimos en que las prácticas políticas clientelares constituyen una limitación para el ejercicio de formas democráticas más igualitarias, entonces resulta indispensable comprender de qué manera los actores articulan estas prácticas con su subjetividad y su cosmovisión. Es que, si no se comprende su fundamento y su sentido, será más difícil diseñar y poner en práctica políticas públicas transformadoras que escapen de las formas clientelares que tienden a capturarlas cuando son ejecutadas a nivel territorial.

En consecuencia, el presente artículo se propone aportar a la comprensión de las prácticas clientelares a nivel local y las dinámicas comunitarias que se articulan con ellas, analizando la búsqueda de asistencia como estrategia de supervivencia del campesinado a partir de un estudio de caso realizado en la provincia de Formosa, Argentina. En concreto, en el artículo se describen los distintos tipos de ayudas disponibles, las formas empleadas por la comunidad para buscar favores y asistencia, los esquemas subjetivos por medio de los cuales se legitiman las ayudas y los sentimientos y afectos en que se apoyan estas prácticas.

\section{Metodología de la investigación y marco teórico}

Se realizó un estudio de caso en una localidad campesina caracterizada por los altos índices de pobreza y por el predominio de productores minifundistas, la cual se encuentra ubicada en la provincia de Formosa, región noreste de la Argentina. Los resultados que aquí se presentan se enmarcan en un estudio más amplio que corresponde a la tesis doctoral del autor, la cual se ocupó de indagar los factores psicosociales que influyen en el desarrollo rural en poblaciones campesinas, ubicándose la dimensión subjetiva del clientelismo político como uno de los ejes de mayor interés. La investigación se apoyó en la realización de observación participante, conviviendo por más de cinco meses con una familia campesina en varios viajes sucesivos, y la toma de entrevistas, 71 a campesinos y 11 a otros actores, incluyendo medianos productores, agentes de desarrollo y un dirigente campesino. De las 71 entrevistas realizadas a campesinos, sólo en 26 
de ellas se abordaron de manera directa las prácticas políticas locales y las estrategias de búsqueda de asistencia. No obstante, en la mayor parte de los encuentros surgieron comentarios y reflexiones de interés que sirvieron para contextualizar la temática. Finalmente, para sistematizar los datos se realizó un análisis de contenido de las transcripciones de las entrevistas y de los registros de observación participante con el apoyo del software para análisis cualitativo Atlas Ti.

A nivel conceptual, el presente trabajo se apoya en los principios teóricos de la psicología comunitaria latinoamericana que considera a las personas como sujetos activos, capaces de transformar su propia realidad (Montero, 1994), a diferencia de los enfoques que privilegian la determinación de las estructuras por sobre la capacidad de los actores. Igualmente, partiendo del objetivo de comprender la manera en que los campesinos perciben y dan sentido a ciertos aspectos de su realidad social, el presente trabajo se apoya en lo que ha sido denominado "abordaje orientado al actor” o “actor-oriented-approach” (Long, 2001), perspectiva que puede ser concretizada desde la psicología tomando las contribuciones del construccionismo social, el cual afirma que lo que percibimos como "realidad” no es más que el resultado de un proceso de construcción social (Berger y Luckmann, 1972; Gergen, 1996).

Por otra parte, los desarrollos sobre clientelismo político también resultan de gran interés para enmarcan el presente trabajo. Así, se parte de definir al clientelismo como un tipo particular de relación de intercambio entre dos sujetos, "en la que existe un patrón y un cliente: el patrón proporciona bienes materiales, protección y acceso a recursos diversos y el cliente ofrece a cambio servicios personales, lealtad, apoyo político o votos” (Audelo Cruz, 2004: 127). De esta manera, en términos generales puede decirse que el clientelismo político implica un vínculo de carácter asimétrico (Auyero, 1997a) articulado a partir de relaciones cara a cara (Trotta, 2003) donde se produce un flujo de bienes, servicios y compromiso de protección, de parte del patrón (Clark, 2004) y de lealtad y apoyo político de parte del cliente. No obstante, también cabe aclarar que la diversidad de formas en que este vínculo se concretiza hace difícil diferenciar de manera absoluta entre política clientelar y no clientelar (Gay, 1997).

\section{Necesidades campesinas y estrategias de subsistencia}

Las unidades campesinas, para subsistir, se ven en la necesidad de cumplir con dos funciones interdependientes: la satisfacción de las necesidades de los integrantes de la familia y la gestión de la producción, para lo que llevan adelante un conjunto de actividades y aplican una variedad de recursos que tienen a su disposición. En términos generales, las necesidades de la familia campesina incluyen alimentación, ropa y calzado, y gastos vinculados con la salud y la asistencia de los niños a la escuela. Por su parte, para gestionar la actividad productiva estos pequeños productores tienen que contar con tierra, con herramientas o recursos para preparar el 
suelo y realizar la siembra, con mano de obra para múltiples tareas, destacándose el raleo y la cosecha, y con insumos como semillas y diferentes tipos de fitosanitarios.

Como es usual en las economías campesinas, centradas en el uso de mano de obra familiar (Manzanal, 1993), buena parte de los requerimientos mencionados anteriormente son cubiertos con el trabajo de los miembros de la familia y con producción de autoconsumo (de la Barra y Holmberg, 2000; Craviotti y Soverna, 1999), lo que incluye vegetales como mandioca y maíz, y subproductos animales como carne, leche y huevos. No obstante, cambios de hábitos en estas economías de subsistencia están llevando progresivamente a la reducción de la producción de autoconsumo y a la reorientación de la producción hacia el mercado (Silvetti y Cáceres, 1998). Por esto, hoy es indudable que en la zona de estudio, y en la mayor parte de las áreas campesinas de la Argentina, los cultivos de renta, en este caso algodón y hortalizas, ocupan un lugar fundamental en la economía familiar. En concreto, la venta de estos productores hace posible obtener recursos monetarios concentrados una o más veces al año, lo que permite enfrentar necesidades que involucran erogaciones de dinero contextualmente significativas como preparación de suelo, compra de insumos, adquisición de ropa y calzado y pago de deudas. No obstante, estos ingresos concentrados, no permiten a los campesinos cubrir los costos de los alimentos que no se obtienen vía autoconsumo, así como pequeños gastos cotidianos que suelen surgir. De esta manera, cobran gran interés los ingresos que se obtienen por la venta de productos de huerta o granja al por menor y la disponibilidad de ayudas públicas como preparación de suelo a bajo costo, entrega de semillas de algodón y planes sociales que proveen mensualmente de dinero en efectivo. Interesante destacar la amplitud de los planes sociales, que según estimaciones propias en base a datos firmes durante el año 2005 habrían alcanzado a aproximadamente un 60\% de las familias campesinas del lugar.

Cabe mencionar que el campesino no acostumbra acumular o mantener dinero en efectivo como forma de ahorro, sino que prefiere hacerlo por medio de bienes más tangibles, como la compra de ganado, de herramientas o la implementación de mejoras en las viviendas o predios (Patiño, 2000). En efecto, trabajos de investigación realizados muestran que los campesinos poseen vínculos y capacidades personales que les permiten manejarse en contextos de escasez de dinero, no de abundancia relativa (Landini, 2011). Esto se observa con claridad cuando se toma conciencia de que estos pequeños productores tienden a utilizar de manera casi inmediata el dinero que reciben por ventas en cantidad para pagar deudas, comprar algún freezer para las viviendas, adquirir insumos para la producción o ahorrar por medio de ganado mayor, quedándose rápidamente sin efectivo. Así, pasado un breve tiempo después de la venta quedan en una posición donde tienen poco margen para responder a imprevistos o, incluso, para asumir los gastos que implica iniciar la actividad productiva. Entonces, es posible que necesiten recurrir a créditos en los almacenes para hacerse de comida y buscar ayuda de manos de patrones para conseguir pre- 
paración de suelo o para comprar insumos, lo que los llevará a quedar obligados con ellos a la hora de vender el producto. Es que los excedentes que obtienen los productores pocas veces les permiten gestionar el ciclo productivo por sus propios medios, quedando siempre expuestos frente a imprevistos climáticos, como sequías y plagas, y personales, como enfermedades y accidentes. Y esta situación, agravada en el caso de los productores más pequeños y de los jornaleros sin tierra, que generalmente viven al día y no tienen siguiera el resguardo de animales vacunos para vender en tiempos de necesidad.

Ahora bien, es en este contexto de pobreza, de falta de reservas y de privaciones materiales que el ir a pedir ayuda o asistencia directa y generalmente personalizada a políticos, funcionarios o productores consolidados, se convierte en una estrategia de supervivencia privilegiada, ante la falta de alternativas, lo que se ve favorecido por múltiples factores. En primer lugar, el hecho de que, como se señaló anteriormente, en las economías campesinas los sujetos más desprotegidos asumen que los actores con mayor poder económico tienen el deber moral de ser generosos con quienes más lo necesitan. En segundo lugar, en la zona existe una amplia gama de proyectos y programas sociales, lo que convierte a las ayudas públicas en algo que forma parte de la cotidianeidad. Además, los campesinos conocen de primera mano que dar ayudas, en gran medida discrecionales, es algo que todos los políticos hacen en tiempos de elecciones, por lo que resulta intuitivo buscar su apoyo en momentos de particular necesidad. En efecto, como los mismos campesinos narran, durante las elecciones los representantes de los distintos partidos suelen acercarse a las casas para preguntar por las necesidades y ofrecer ayudas, comprometiéndose muchas veces a entregarlas antes de las elecciones. Por otra parte, también cabe señalar que la necesidad de buscar ayudas en políticos y patrones se hace más imperiosa cuando se toma conciencia de que las redes de ayuda mutua, formadas por pares y vecinos, se encuentran sometidas a similares carencias, por lo que pocas veces resultan apropiadas para resolver los problemas que puedan presentarse. Finalmente, también corresponde mencionar lo indicado en el párrafo anterior, que en la economía campesina los pequeños productores no cuentan con capacidades para gestionar dinero en efectivo, por lo que tienden a enfrentarse a situaciones de escasez con gran facilitad.

Así, se observa que, por diferentes razones, la búsqueda de ayudas o de asistencia directa constituye una necesidad para muchos campesinos. Sin embargo, al estar vaciadas sus redes de ayuda mutua y al no poseer ahorros, se hace necesario recurrir a quienes están situados en una mejor posición socioeconómica o a quienes controlan recursos públicos que puedan dar respuesta a estas necesidades.

\section{Ayudas disponibles y formas de buscar asistencia}

Partiendo de la investigación realizada puede afirmarse que los pedidos de ayuda a políticos y patrones poseen dos formas básicas que se 
entrelazan como un continuo, contraponiéndose ambas a aquellos beneficios asegurados en términos de derechos universales efectivos, como pueden ser el acceso a la salud pública o a la educación primaria y secundaria. El primer polo de este continuo está referido a todas aquellas ayudas instituidas o establecidas localmente, como por ejemplo la preparación de suelo para la siembra por parte del municipio a bajo costo, la cual está ampliamente extendida para el sector campesino. En este caso, como señala un entrevistado, "a todos le hacen. Un poco tenés que ir a chillarle nomás, tenés que ir a llorarle, eso nomás [...] Si vos no sos de ellos [de su partido político] tenés que ir, igual tenés que ir a llorarle”. Es decir, se trata de ayudas relativamente establecidas que es necesario ir a pedir para conseguirlas, ya sea con mayor o menor insistencia, pero que no son entregadas de por sí ni tenidas como derechos instituidos. De esta forma, al tener que ser requeridas en un contexto cara a cara, se alejan de las pautas formales e impersonales que caracterizan al estado burocrático y se convierten en ayudas personalizadas, que si bien no llegan a establecerse como favores personales, al menos inducen la construcción de un vínculo jerárquico de asistencia entre actores desiguales. Dentro de esta categoría, tal vez sea posible agregar la distribución de medicinas en los edificios municipales a aquellos pobladores que están incorporados al Programa de Asistencia Integral al Pequeño Productores Agropecuario (PAIPPA), y la entrega de semillas de algodón y de comestibles por parte de diferentes programas públicos.

En el segundo polo, aparecen necesidades a las cuales no dan respuesta las ayudas institucionalizadas relativamente universales como las mencionadas. Puede ser que una madre busque llevar a su hijo para que sea atendido en un hospital fuera de la provincia y necesite pasajes de micro, que alguien desee obtener un trabajo o un plan social, una bolsa con mercadería, materiales para hacer una vivienda o incluso busque dinero para pagar carpidores o semillas de hortalizas para poder plantar. Los contenidos de estos pedidos (y las necesidades a las que apuntan) pueden ser múltiples, ya que hacen referencia a una diversidad de situaciones y contextos. Pero, en cualquier caso, se expresan, como señala Auyero (1997b), en el acercamiento del cliente al mediador en el momento en que surgen problemas que requieren de un favor especial.

En el caso investigado, además de pedir a distintos funcionarios u operadores políticos, los campesinos también pueden recurrir a productores agropecuarios consolidados (denominados por la gente "patrones”). Acercarse a un político o a un productor estructurado para pedir ayuda, está usualmente vinculado con el tipo de problema a resolver o de ayuda a obtener. Para buscar planes sociales, empleos públicos o bolsas de mercadería, sólo es posible contactarse con actores vinculados al ámbito político. Por su parte, la necesidad de semillas de hortalizas, de venenos o de adelanto en efectivo para la carpida o raleo, usualmente requiere del acercamiento a un productor que pueda oficiar de patrón, al que luego deberá venderse la producción que se obtenga. Finalmente, la búsqueda de dinero frente a situaciones de emergencia o de marcada necesidad puede, potencialmente, ser resuelta por ambos, aunque en 
distintas condiciones. En el caso de patrones a cuenta de la cosecha, y en el de los políticos, a partir de la existencia de vínculos con la facción del operador al que se contacta.

Estos requerimientos de ayudas especiales poseen dos características esenciales. La primera, que es necesario ir a pedirlos para conseguirlos. Es decir, no se entregan u ofrecen de manera genérica, espontánea o universal, como sucede en el caso de la educación pública o los hospitales financiados por el gobierno. La segunda, y aún de mayor interés, es que se trata de solicitudes que pueden ser rechazadas, es decir, que pueden no ser satisfechas por quien tiene capacidad o poder para cumplir con ellas. Respecto de esto, es claro que existe una diferencia entre ambos polos mencionados. En el caso de las ayudas institucionalizadas pero no universalizadas en términos de derechos, la falta de cumplimiento refiere a una demora en el tiempo. Si bien se las termina obteniendo cuando se las pide, esto puede retrasarse, con el consiguiente perjuicio para el agricultor, como sucede si la preparación de suelo a bajo costo recién se obtiene luego de los plazos óptimos para cultivar. Por su parte, y mientras más se aleja uno del polo de estas ayudas, se llega a otras que pueden no ser resueltas en absoluto, dependiendo de la voluntad de quien controla los recursos que se necesitan.

Estos pedidos de ayudas o favores especiales pueden clasificarse en tres categorías. La primera puede denominarse "pedido-reclamo", la segunda "pedido formal-grupal" y, finalmente, el "pedido personalizado", de mayor importancia tanto cuantitativa como psicosocial. El pedido-reclamo, precisamente, expresa el movimiento activo de los campesinos orientado a exigir la satisfacción de determinadas necesidades, presionando o confrontando con las autoridades o el gobierno. Esta modalidad se basa en la existencia de un grupo u organización social (que puede ser desde un Movimiento Campesino de nivel provincial hasta una pequeña agrupación de productores) que es el que lleva adelante el reclamo. Dado que parte del requerimiento de un grupo, el pedido-reclamo suele orientarse a demandas colectivas o a satisfacer necesidades comunes como obtener semillas, arreglar una escuela venida a menos o, simplemente, buscar atención para los problemas del hombre de campo. Este tipo de pedido se sostiene en el uso de la fuerza provista por la agrupación (o de la amenaza de ello). Como señaló un campesino: "porque ellos [la organización] hacen un proyecto, presentan un proyecto. Eso van y lo elevan al gobierno, entonces dicen 'esto nosotros queremos' [...] y les hacen corte, porque si no, no les dan la respuesta que ellos quieren, cortan la ruta, le hacen protesta”.

El pedido formal-grupal corresponde a uno efectuado por un grupo de personas que posee un problema común. Puede tratarse de la solicitud de guardapolvos para alumnos de la escuela, de la necesidad de conseguir luz para viviendas rurales o de hacer un puente para facilitar el cruce de un pequeño río. El pedido efectuado puede ser escrito (y por tanto formalizado) o simplemente realizado por medio de un represen- 
tante. Aquí hay que resaltar que pese a tratarse de un grupo de personas, éstas suelen ser menos que las necesarias para generar un pedido-reclamo y que, en todo caso, son más una colección de individuos que comparten un problema que un grupo. Así, pueden ser productores de cierto pueblo que votan en un determinado municipio o grupos de padres o vecinos de una determinada colonia. Aquí, en términos generales, no hay voluntad ni de construir una asociación permanente ni de entrar en confrontación con el gobierno, sino que se busca apelar a las razones, a la descripción de la necesidad o a la negociación, como vías para obtener una respuesta favorable. Como ejemplo puede comentarse el caso de un grupo de productores que aún no cuenta con la tenencia de la tierra en la que trabaja: "hicimos una nota, firmamos todos acá y yo llevé eso en el PAIPPA, en Formosa. Y ellos me dijeron que se van a encargar de hacer las gestiones, para que vengan y se mensure y nos hagan el título provisorio”. Finalmente nótese que tanto el pedido-reclamo como el pedido formal-grupal son formas de ir a pedir que solamente se dirigen a las autoridades municipales o provinciales, pero nunca a operadores políticos sin cargo o a patrones, como sí sucede en el caso siguiente.

El pedido personalizado es el tercer modo de ir a pedir que ha sido identificado. Es, a la vez, el más mencionado por los entrevistados. Se trata de solicitudes realizadas de manera personal por quien detenta un problema ante quien puede solucionarlo o puede realizar la gestión para ello. Por esto mismo, más que al bien común o al bien grupal, estos pedidos se encuentran orientados a responder a necesidades individuales 0 familiares. Además, si bien tanto en el pedido-reclamo como en el formalgrupal existe la posibilidad de que la ayuda no sea concedida, en este caso las consecuencias de este hecho suelen ser mucho más graves a nivel personal. En el pedido-reclamo, las personas se acercan a sus interlocutores de manera activa y confrontativa, percibiendo en el proceso su propia fuerza. En el pedido formal-grupal los objetivos son beneficios compartidos que usualmente no responden a necesidades acuciantes o decisivas. Sin embargo, a diferencia de los anteriores, en el pedido personalizado los peticionantes muchas veces dependen fuertemente de los resultados de la gestión. Si no obtienen los medicamentos que necesitan ¿qué será del enfermo?, si no se logra el empleo, la bolsa de comida o el plan social buscado ¿cómo se alimentará a la familia?, si no se consigue el dinero para compra de semillas de hortaliza o para insecticidas, ¿en qué se trabajará durante la campaña agrícola? Aquí, efectivamente, las personas perciben su dependencia respecto de aquellos que tienen en sus manos ayudarlos. Asimismo, se sienten (y describen), muchas veces, como pobrecitos, necesitados, y aun, inermes. De hecho, se encuentran en una situación de desprotección en la cual no pueden gestionar por sí mismos la resolución de sus problemas por lo que están a merced de quien podría negarse a ayudarlos. Esto muestra la radicalidad de la experiencia de necesidad, situación que permite intuir las consecuencias afectivas que generarán tanto las respuestas positivas como las negativas al pedido de ayuda por parte del campesino. 


\section{Procedimiento y legitimidad subjetiva del pedido personalizado}

El pedido personalizado posee una forma propia, unos procedimientos relativamente estandarizados y unas representaciones específicas que lo acompañan. Como se señaló anteriormente, el primer momento es el hecho mismo de ir a buscar a quien puede ayudar. Dado que ya finalizó el período electoral, los políticos ya no pasan por las casas sino que, como dicen los entrevistados, "esperan en la oficina [...] que se vaya uno y le hable a ellos”. Así, hay que ir, hay que acercarse a donde están ellos, haciendo fila si fuera necesario, y estando dispuestos a esperar. Este ir a pedir es comprendido por muchos entrevistados como un "molestarle" o "hincharle" a los políticos. En este sentido, atender a las personas para estas cuestiones no aparece, al menos aquí, como algo propio del cargo o función que éstos ejercen, en el caso de ser funcionarios públicos, sino como algo que va más allá y que implica "una molestia”. Es por eso que los entrevistados aclaran que no hay que estar "molestando" todo el tiempo a los posibles benefactores: "hay momentos que tenés que molestar y hay momentos que no”. Por otra parte, como es necesario ir, esto crea un inconveniente para los campesinos, principalmente para aquellos que viven más lejos del pueblo, porque el desplazarse en sí mismo representa un problema: "llegar ahí ya te cuesta”. Más cuando los problemas o cuestiones no se resuelven en el momento y va a ser necesario volver otras veces. De hecho, aunque todos hablan de este "ir a pedir" a los políticos, parte de los entrevistados señala que no ha ido nunca.

Una vez que se ha accedido a hablar, uno debe contarle a quien lo atiende cuál es su necesidad o su problema: "tenés que ir a llorar allá", "vos te vas le decís que te ayude para autoconsumo, que no tenés nada, que tenés muchas criaturas". Y esto, no buscando ser estrictamente objetivos al describir la necesidad sino procurando presentar una imagen de sí mismos que insista en la situación de pobreza, sufrimiento y desprotección. Como señala Goffman "dondequiera que hay un test de medios [económicos] es probable que haya una exhibición de pobreza”. (1971: 51). Así, el procedimiento requiere plantear qué es lo que uno quiere para solucionar su situación formulando un pedido. Incluso, algunos agregan que se puede proponer reciprocar el favor diciendo, por ejemplo, que uno se compromete con el voto. En este contexto, pareciera que lo usual no es obtener una respuesta inmediata sino que muchas veces va a ser necesario insistir, lo que lo hace aun más difícil para quienes viven alejados en el campo o en las colonias campesinas. Sin embargo, hay que estar dispuestos a volver porque es común que quien escucha el pedido pida al campesino que vuelva al día siguiente, aunque esto no es ninguna garantía: "por ahí te dicen 'vení mañana' y te vas y no pasa nada otra vez”. De hecho, esta estrategia parece orientada, muchas veces, a no responder directamente de manera negativa, posponiendo la definición para los días por venir, apostando al cansancio del campesino. De todas formas, en situaciones de urgencia, como en el caso de un accidente o de la muerte de un familiar cercano, lo usual es ser ayudados. 
Ahora bien, ¿cómo justifica el campesino la legitimidad del pedido que hace?, ¿por qué resulta adecuado o correcto, a sus ojos, pedir ayuda a funcionarios públicos, e incluso a otros actores pudientes como intermediarios o patrones, de manera personalizada? Son varios los argumentos implícitos que pueden encontrarse en las palabras de los entrevistados, aunque prácticamente ninguno de ellos refiere a la existencia de derechos básicos que el gobierno deba garantizar. Respecto de ayudas como preparación de tierra, semillas o aun empleos públicos, se suele destacar que lo que se busca es una oportunidad de trabajar. Así, se justifica el pedido porque lo que uno pretende es algo legítimo: poder trabajar y esforzarse, uno no es un vago, quiere trabajar y por eso debería ser ayudado. Como plantea una campesina:

Y lo que yo le pedí a la intendenta hace tiempo [es] que me dé un sueldo [...] porque yo no tengo la costumbre de ir a sentarme en la municipalidad hasta las 11 de la mañana esperando que me den un vale de 1 kilo de carne [...]. Voy a trabajar hasta que me caiga a morir.

Sin embargo, la percepción mayoritaria es que el justificativo o la razón que legitima el pedido no es más que la combinación de la imposibilidad de resolver la necesidad por uno mismo y la disponibilidad de los recursos por parte de aquel a quien se requiere la ayuda. Como se señaló, en las economías campesinas se espera que los productores más acaudalados sean generosos y aporten a la satisfacción de las necesidades de aquellos que tienen un mal pasar, a partir del principio moral de que todos tienen derecho a subsistir (Henningsen, 2001).

La respuesta que los campesinos dicen recibir ante estos pedidos es dispar. Algunos señalan que, en ciertas oportunidades, se pueden tener respuestas positivas, no así en otras, según la suerte. Sin embargo, existen dos marcos usados para interpretar estas situaciones. El primero explica la ayuda o su falta recurriendo a la bondad del político en cuestión: "algunas personas digamos que se van y le piden esto al intendente y capaz que le dice 'no, no te vamos a poder hacer' y hay algunos que son buenos, te vas y le decís ‘bueno, ¿me podés ayudar con esto?', ‘sí, te dice’”. De esta forma, sería una cualidad humana propia de quien da la que explicaría el sí o el no. En contraposición, un segundo esquema interpretativo sostiene que el recibir una respuesta positiva a lo que se necesita o se pide tiene que ver más con una sectorización de las prácticas políticas. Así, cada partido o línea política sólo ayudaría a sus propios partidarios, siendo la fuerza gobernante la que controla mayor cantidad de recursos para hacerlo. Sin embargo, esta última explicación no podría dar cuenta de por qué las personas, aun así, piden a aquellos que ya saben que no van a darles por ser de otro partido o línea política. De todas formas, hay que reconocer que los límites entre adherentes de uno y otro partido a veces son difusos y, a la vez, puede haber cambios de posicionamiento a raíz de las ayudas, ya que la posición de cada actor siempre está sujeta a reacomodamientos. 
En cualquier caso, la percepción mayoritaria sostiene que las ayudas concedidas en el contexto de estos pedidos no son adecuadas ni suficientes para satisfacer las necesidades, habiendo un déficit en la generosidad de quienes pueden ayudar. Además, muchas veces, los compromisos no parecen llegar a cumplirse. De esta forma, el comprometerse a ayudar y no cumplir o el pedir al "cliente" que vuelva en unos días son, muchas veces, formas políticamente correctas de decir que no. En otras oportunidades, no se trata de que no respondan sino que lo que dan no es suficiente para solucionar los problemas planteados: "porque ¿qué te van a dar los políticos? se enferma tu hijo, estás por hacerlo pasar [trasladar] a otra localidad, te vas, le pedís a los políticos, te van a dar 10, 20 pesos, y con eso no solucionás vos”.

\section{Trasfondo afectivo de las ayudas personalizadas}

Finalmente, es necesario destacar la vinculación afectiva que se genera (o consolida) en las situaciones en las cuales estas ayudas son dadas, lo cual es fuente de lealtades y de prácticas de reciprocidad. Pero para hacer esto, es conveniente tener en cuenta dos elementos que configuran el contexto y el modo en que la relación se produce. El primero, la situación de profunda desprotección, inseguridad y dependencia en que se pueden encontrar las personas sujetas a necesidades reales que no pueden ser resueltas sin la ayuda de otro que, de hecho, puede negarse a ayudar. El segundo, el hecho de que "la dinámica del clientelismo [...] se basa en una relación personalizada e individual entre un patrón y su cliente” (Pfoh, 2005: 136), razón que llevará a los campesinos a tender a explicar las decisiones tomadas por los políticos o patrones, en los momentos en que se producen, a partir de la buena o mala voluntad de las personas y no de factores ajenos a la situación cara a cara. Así, el "ir a pedir” (en su modalidad de pedido personalizado) se encontrará enmarcado por la experiencia de dependencia y desprotección del pequeño productor y por la existencia de un otro poderoso que puede o no decidir ayudar según los designios de su voluntad. Consecuentemente, podrán generarse fuertes sentimientos tanto de repudio y rechazo como de aprecio y gratitud, según sea la respuesta y la interpretación de los motivos de quien ayuda. De esta forma, será el agradecimiento la emoción predominante cuando la asistencia sea percibida como resultado de la bondad o de la preocupación del político por las necesidades de los pobres y no como fruto de un interés electoral. No obstante, la gratitud en este tipo de situaciones no es algo que deba esperarse sólo del campesino. Al contrario, el mismo investigador se encontró en una situación que le provocó la misma emoción cuando un campesino que no era su amigo, un mediodía de verano con una temperatura cercana a los $40^{\circ}$, le dio una botella de agua fría de manera inesperada pero sumamente oportuna. En este sentido, la gratitud es un sentimiento humano, lo que sucede es que dada la situación de desprotección y las desigualdades existentes en Misión Tacaaglé, pareciera ser que se trata de experiencias profundas y frecuentes, con una importante potencialidad para estructurar vínculos interpersonales jerárquicos. 
La gratitud en tanto sentimiento aparece como la reciprocidad frente al favor gratuito recibido. Si el agua hubiera sido comprada con dinero o si las chapas hubieran sido pagadas con votos, el vínculo se hubiera extinguido cuando las partes hubieran equiparado su cuenta. Sin embargo, la percepción de gratuidad en la ayuda o el favor recibido genera algo del orden de lo impagable ya que implica que no puede ser devuelto. Lo gratuito no es lo que no vale nada sino lo que no tiene precio, por eso, la única forma de pago, o al menos de equiparación, es la gratitud, la que aquí, en este contexto, se encuentra fortalecida tanto por la profunda situación de necesidad y dependencia de quien pide como por la personalización mediante la que se estructura la entrega de ayudas. Así, la gratitud es comprendida en este sentido como la respuesta a una deuda que no se puede pagar, por lo que se tiende a perdurar a lo largo del tiempo:

Pienso que no voy a pagar nunca... todos me dicen 'no, ya con tu voto pagaste', no, no es así, sea que mi manera de ser es muy distinta a los otros, yo pienso que lo que él [un operador político de la zona] hizo por mí no lo voy a pagar a nadie, nunca, por más votos que le dé. El agradecimiento [es] lo que está por delante.

Claro que el vínculo tiene que ser actuado y reproducido a partir de contactos frecuentes para mantenerse en el tiempo, pero son los propios efectos de estos sentimientos los que inducen a tales interacciones posteriores. En este contexto, cobra cabal sentido la devolución de los favores recibidos con votos o apoyo político, en tanto se trata de un intento (siempre fallido) por devolver lo gratuito, es decir, por equiparar los favores prestados. Obviamente, todo esto no significa que la gratitud sea el sentimiento que está en la base de todo vínculo clientelar. De hecho, existen relaciones mucho más instrumentales y sin dudas numerosos casos pueden ser mejor explicados aduciendo vínculos de opresión en lugar de relaciones afectivas. Pero desconocer la existencia de éstas junto con las prácticas clientelares más descarnadas no es más que una simplificación excesiva de un fenómeno complejo.

\section{Reflexiones finales}

En el presente trabajo, se abordó la búsqueda de asistencia como estrategia de supervivencia en una comunidad campesina y su articulación con prácticas clientelares. En primer lugar, se analizaron las necesidades que deben satisfacer las familias de pequeños agricultores y las fuentes de recursos que tienen a su disposición, destacándose el hecho de que, dada su situación de pobreza, el vaciamiento de las redes de apoyo y la falta de capacidad personal para gestionar dinero en efectivo, es usual que los campesinos necesiten recurrir a actores más pudientes tanto para responder a necesidades puntuales como para encarar el ciclo productivo.

Por otra parte, también se construyó una clasificación de los tipos de ayudas existentes y de las modalidades por medio de las cuales los cam- 
pesinos procuran acceder a ellas. Así, se contrastó la asistencia pública universalizada (como el acceso a la educación y a la salud pública), con las ayudas relativamente instituidas a nivel local (como la preparación municipal de suelo a bajo costo) y otras ayudas no estandarizadas que corresponden a la diversidad de problemas con que se pueden enfrentar las personas en sus vidas. Igualmente, se construyó una clasificación de las distintas modalidades por medio de las cuales se pueden buscar estas ayudas, particularmente las últimas, haciéndose referencia a un pedido-reclamo, a uno formal-grupal y a uno personalizado. Interesante mencionar que estas ayudas, al tener que ir a pedirse en situaciones cara a cara, se alejan de la despersonalización con la que el estado burocrático suele responder a las necesidades de los más desprotegidos. Siguiendo los comentarios de Güne_Ayata (1997), cabe destacar aquí uno de los valores que tienen para el campesino las prácticas de patronazgo o de tipo clientelar: que se trata de sistemas donde las personas y sus necesidades no son tratadas con indiferencia por el sistema sino de manera humana y personalizada.

Después, se abordó el procedimiento utilizado para solicitar las ayudas, destacándose la importancia que cobra la modalidad de presentación de sí mismo como una persona trabajadora y esforzada que se encuentra en situación de verdadera necesidad, con el fin de maximizar la probabilidad de recibir asistencia. No obstante, esto no debe llevarnos a pensar que se trata, al menos en la mayoría de los casos, de una estrategia conciente y voluntaria de manipulación. Por el contrario, existen aspectos situacionales que tienden a activar las dimensiones del sí mismo que se condicen con el modo en que los campesinos se presentan ante quienes pueden ayudarlos (Landini, 2010). Como señala Goffman (1971), en situaciones como la descripta las más de las veces los actores se sienten comprometidos subjetivamente con la imagen que presentan de sí mismos en la interacción.

Por otra parte, también se abordaron las representaciones por medio de las cuales los campesinos dan sentido y legitimidad a la búsqueda de ayudas personalizadas en funcionarios, referentes políticos y patrones. Se trata, concretamente, de un principio que postula que todas las personas tienen derecho a vivir con dignidad, razón por la cual aquellos que tienen más de lo que necesitan tienen la obligación moral de ayudar a quienes menos tienen. Esta regla, humanamente muy justa, indudablemente se aleja de los principios de la economía capitalista en la que estamos acostumbrados a manejarnos, la cual, sin lugar a dudas, tiende a dar mayor valor al derecho a la propiedad privada que a las necesidades de las personas.

Asimismo, también cabe destacar la dimensión afectiva que muchas veces estructura las prácticas de reciprocidad que se dan entre actores desiguales, en este caso campesinos pobres y actores que disponen o controlan recursos que pueden responder a sus necesidades. Es que muchas veces el clientelismo ha sido visto exclusivamente como una forma de dominación, cuando aquí también aparece sostenido por vínculos interpersonales que se apoyan en sentimientos de gratitud. Así, el esquema que interpreta al clientelismo como un intercambio descarnado de bienes por votos, debería 
ampliarse para admitir la posibilidad de que sea la gratitud generada por las ayudas lo que luego se exprese en apoyo político, como forma de reciprocidad, y no a la inversa. Además, dado que la gratitud, fundada en la percepción de la ayuda gratuita recibida en tiempos de necesidad, no cuantifica el valor de los bienes y apoyos que se intercambian, el vínculo que genera se caracteriza por no agotarse cuando los favores son retornados, lo que sí tiende a suceder cuando existe un intercambio estricto de ayudas por votos. En definitiva, resulta obvio que existen casos en los cuales la mejor explicación de ciertos vínculos clientelares es el intercambio directo de favores por votos. No obstante, esto no debe hacernos olvidar la dimensión afectiva que muchas veces estructura estos vínculos, para poder estudiarlos en su diversidad.

Para finalizar, cabe aclarar que la búsqueda de asistencia y de favores en actores pudientes no debe ser identificada así y sin más con clientelismo político. De hecho, se trata de una modalidad comunitaria de gestión de las necesidades que abarca mucho más que prácticas políticas clientelares. De hecho, agentes de desarrollo rural ajenos a dinámicas partidarias, comerciantes de pueblo y productores consolidados también forman parte de las personas a las cuales, en ciertos contextos, se puede recurrir para buscar ayuda y favores. Incluso, al mismo autor de este trabajo, en diferentes oportunidades, se le pidió que comprara juguetes para los niños o que aportara para la fiesta de una joven que cumplía 15 años. Así, queda claro que se trata de un modo de funcionamiento de este tipo de comunidades que sólo se convierte en clientelismo político cuando se articula con la lógica de acumulación de poder de ciertos partidos o sectores que aprovechan de estas dinámicas para alcanzar sus propios fines. Por esto fue que, durante el artículo, si bien se hizo referencia al clientelismo, también se indicó que se trata de prácticas que no son en sí clientelismo sino que se ubican en su base. En definitiva, se trata de un fenómeno complejo que es necesario comprender con mayor profundidad para generar e implementar políticas públicas que, asumiendo la existencia de estas prácticas, eviten ser incorporadas a lógicas que no buscan el bien de la gente sino la acumulación de poder en ciertos sectores o grupos minoritarios de la sociedad. 


\section{Nota}

${ }^{*}$ El presente artículo fue realizado con el apoyo de una beca de postgrado del Consejo Nacional de Investigaciones Científicas y Técnicas (CONICET) de la Argentina y forma parte de la investigación doctoral del autor. 


\section{Bibliografía}

Audelo Cruz, J. (2004), “¿Qué es el clientelismo? Algunas claves para comprender la política en los países en vías de consolidación democrática”, en Estudios Sociales, vol. 12, № 24, Hermosillo, pp. 124-142, México.

Auyero, J. (1997a), “Introducción”, en J. Auyero (Comp.), ¿Favores por votos? Estudios sobre clientelismo político contemporáneo (pp. 13-99), Losada, Buenos Aires.

Ídem (1997b), “Evita como performance. Mediación y solución de problemas entre los pobres urbanos del Gran Buenos Aires”, en J. Auyero (Comp.), ¿Favores por votos? Estudios sobre clientelismo político contemporáneo (pp. 167-232), Losada, Buenos Aires.

Ídem (1999). “'From the client's point(s) of view': how poor people perceive and evaluate political clientelism”, en Theory and Society, vol. 28, $\mathrm{N}^{\circ} 2$, pp. 297-334, Holanda.

Ídem (2000), “The logic of clientelism in Argentina. An ethonographic account”, en Latin American Research Review, vol. 35, N 3, pp 55-81, Montreal,.

Ídem (2001), La política de los pobres. Las prácticas clientelares del peronismo, Manantial, Buenos Aires.

Berger, P. y Luckmann, T. (1972), La construcción social de la realidad, Amorrortu, Buenos Aires.

Clark, J. (2004), "Social movement theory and patron-clientelism. Islamic social institutions and the middle class in Egypt, Jordan, and Yemen”, en Comparative Political Estudies, vol. 37, Nº 8, pp. 941-968.

Craviotti, C. y Soverna, S. (1999), Sistematización de estudios de casos de pobreza rural, Ministerio de Economía. Secretaría de Agricultura, Ganadería, Pesca y Alimentos. Dirección de Desarrollo Agropecuario. PROINDER, Buenos Aires.

De la Barra, A. y Holmberg, G. (2000), "Productive and economic performance of peasant systems under surplus and subsistence conditions: a case study”, en Agricultura Técnica, vol. 60, N 1, pp. 52-61, Santiago de Chile.

Gay, R. (1997), "Entre el clientelismo y el universalismo. Reflexiones sobre la política popular en el Brasil urbano”, en J. Auyero (Comp.), ¿Favores por votos? Estudios sobre clientelismo político contemporáneo (pp. 65-92), Losada, Buenos Aires.

Gergen, K. (1996), Realidades y relaciones. Aproximaciones a la construcción social, Paidós, Barcelona. 
Goffman, E. (1971), La presentación de la persona en la vida cotidiana, Amorrortu, Buenos Aires.

Güne_-Ayata, A. (1997), “Clientelismo: premoderno, moderno, posmoderno”, en J. Auyero (Comp.), ¿Favores por votos? Estudios sobre clientelismo político contemporáneo (pp. 41-63), Losada, Buenos Aires.

Henningsen P. (2001), "Peasant society and the perception of a moral economy. Redistribution and risk aversion in traditional peasant culture”, en Scandinavian Journal of History, vol. 26, № 4, pp. 271-296.

Landini, F. (2010), Peasant identity. Contributions towards a rural psychology from an Argentinean case study, Manuscrito enviado para publicación.

Ídem (2011), Income and use of money in the peasant economy. Contributions from a case study, Manuscrito enviado para publicación.

Long, N. (2001), Development Sociology. Actor Perspectives, Routledge, Londres.

Manzanal, M. (1993), Estrategias de supervivencia de los pobres rurales, Centro Editor de América Latina, Buenos Aires.

Montero, M. (1994), "Vidas paralelas. Psicología comunitaria en Latinoamérica y en Estados Unidos”, en M. Montero (Coord.), Psicología social comunitaria. Teoría, método y experiencia (pp. 19-46), Universidad de Guadalajara, Guadalajara.

Patiño, J. (2000), "Prácticas y racionalidad productiva. Estrategias de los Mazahuas de Ixtlahuaca”, en Convergencia, año 7, $\mathrm{N}^{\circ} 22$, México, pp. 193-246.

Pfoh, M. (2005), “La formación del Estado Nacional en América Latina y la cuestión del clientelismo político”, en Revista de Historia Americana, No 136, pp. 131-148, México.

Silvetti, F. y Cáceres, D. (1998), “Una perspectiva sociohistórica de las estrategias campesinas del noreste de Córdoba, Argentina”, en Debate Agrario, No 28, pp. 103-127, Lima.

Trotta, M. (2003), Las metamorfosis del clientelismo político. Contribución para el análisis institucional, Espacio Editorial, Buenos Aires.

Recibido: 26.01.2011

Aceptado: 06.06.2012 